\title{
Una Descripción General y Actualizada de Miniplacas y Minitornillos. Efectos Dentoalveolares y Esqueléticos
}

\author{
A General and Updated Description of Miniplates and Miniscrews. \\ Dentoalveolar and Skeletal Effects
}

Francisca Durán'; Francisca Hormazábal²; Ximena Toledo; Ru-Harn Chang; ; Natalia González ${ }^{2} \&$ Paulina Sciaraffia ${ }^{2}$

DURÁN, F.; HORMAZÁBAL, F.; TOLEDO, X.; CHANG, R. H.; GONZÁLEZ, N. \& SCIARAFFIA, P. Una descripción general y actualizada de miniplacas y minitornillos. efectos dentoalveolares y esqueléticos. Int. J. Odontostomat., 14(1):136-146, 2020.

RESUMEN: En ortodoncia, las miniplacas se utilizan como dispositivo de anclaje temporal (TAD) para la realización de movimientos dentales que permiten el uso de fuerzas ortopédicas en ellos. En comparación con los mini tornillos, las miniplacas tienen la ventaja de una tasa de falla muy baja, pero la desventaja es que para la extracción se necesita el mismo acto quirúrgico que se realizó para la instalación. El objetivo de este estudio es realizar una revisión bibliográfica de las indicaciones de miniplacas en pacientes con mordidas abiertas, clase II y anomalías de clase III, y buscar cómo las miniplacas han mejorado los tratamientos de ortodoncia. La información principal se reunió buscando en PubMed con las palabras clave enumeradas a continuación. Afirmamos que las miniplacas están indicadas para la retracción en masa de la arcada, donde se observó que la fuerza de $150 \mathrm{~g}$ aplicada en los molares superiores es suficiente no solo para empujar los molares hacia atrás en una clase I corregida, sino también para iniciar la retracción de premolares, caninos e incisivos. En pacientes con mordida abierta, las miniplacas se definen como un método seguro, una alternativa rápida y menos costosa a la cirugía ortognática. Y en pacientes de las clases II y III se utilizan sin producir efectos dentoalveolares que sustituyan a los dispositivos extraorales como máscaras, con dispositivos intraorales y elásticos (BAMP).

PALABRAS CLAVE: miniplaca de ortodoncia, minitornillo de ortodoncia, dispositivo de anclaje temporal, dispositivo de anclaje óseo, retracción maxilar de anclaje óseo (BAMP), anclaje esquelético.

\section{INTRODUCCIÓN}

Entre los dispositivos, de titanio o sus aleaciones, para anclaje esquelético se pueden destacar tres principales: implantes oseointegrados, mini-implantes y miniplacas (Sekima et al., 2009). Los últimos 2, se encuentran dentro de los no osteointegrados, ya que se diseñan para uso temporal y/o sólo para la fijación de dos segmentos óseos, por lo que el acabado de la superficie lisa y pulida sumado a contaminantes propios de la fabricación, inhiben la oseointegración (Erverdi \& Üsümez, 2010).

Generalidades de las miniplacas. "Miniplaca" es una placa de titanio con agujeros que permiten la colocación de minitornillos para su fijación en el hueso cortical del maxilar o mandíbula.
En Ortodoncia se utilizan como dispositivo de anclaje óseo temporal (TAD) para la realización de algunos movimientos dentarios, teniendo la particularidad de permitir el uso de fuerzas ortopédicas en ellas (Cornelis \& De Clerck, 2007).

Una de las principales ventajas de las miniplacas es que pueden ser colocadas en cualquier parte de la boca, con hueso alveolar y hueso basal. Sin embargo, su inserción involucra un manejo quirúrgico mayor con levantamiento de colgajo, lo que requiere de un cirujano oral (Tsui et al., 2012).

Tasas de éxito, estabilidad y complicaciones. Quizás la mayor ventaja de las miniplacas es su alta tasa de

\footnotetext{
${ }^{1}$ Ivy Meadow Lane. Apt 1H. Durham, NC, USA. 27707.

2 Departamento del Niño y Ortopedia Dento - Maxilar, Escuela de Odontología. Universidad de Chile, Santiago, Chile.
} 
éxito, que según una revisión sistemática del 2012 fluctúa entre el 91,4\% - $100 \%$ (Tsui et al.) y en contraparte, revisiones avalan su baja tasa de falla que fluctúa entre 2,8 \% 7,3 \% (Nagasaka et al., 1999; Choi et al., 2005; Takaki et al., 2010), en comparación con minitornillos, además cuando es necesario mantener el dispositivo por un largo periodo de tiempo (Schätzle et al., 2009).

En aproximadamente $40 \%$ de los casos hay complicaciones, en Tabla I se explica con qué factores se ven relacionadas y cuales son (Faber et al., 2008; Costello et al., 2010; Lama et al., 2018).

En resumen, las miniplacas son preferidas cuando se plantea la aplicación de mayores fuerzas ortodónticas, el movimiento de varios dientes o tiempo de tratamiento más largo. Ventajas y desventajas explicadas en Tabla II.

\section{INDICACIONES}

\section{RETRACCIÓN EN MASA DE LA ARCADA}

Los microtornillos, que generalmente se colocan entre las raíces, pueden limitar la cantidad de distalización posible, en cambio las miniplacas, al estar alejadas de las raíces, permiten la distalización de todo el arco.

Un estudio realizado por Cornelis \& De Clerck, mostraron, a través de superposiciones de modelos digitales, la cantidad de distalización de la arcada superior en pacientes sin crecimiento y los efectos provocados en los maxilares, en donde el arco mandibular no fue tratado se utilizó como referencia (Fig. 1).

Se vio que la fuerza de $150 \mathrm{~g}$ aplicada sobre los molares maxilares es suficiente no sólo para empujar los molares hacia atrás en una posición de Clase I sobre corregida, sino también para iniciar la retracción de los premolares, caninos e incisivos. Sin embargo, en pacientes con acople anterior al inicio, la distalización espontánea del sector anterior fue más restringida (Fig. 2).

Tabla I. Complicaciones de miniplacas y factores relacionados con ellas.

\begin{tabular}{lcc}
\hline Dispositivo & Paciente & Operador \\
\hline Fracturas & Mala calidad ósea & $\begin{array}{c}\text { Mala elección del sitio de } \\
\text { colocación }\end{array}$ \\
Aflojamiento por defecto del diseño & $\begin{array}{l}\text { Higiene } \\
\text { infección de los tejidos circundantes, } \\
\text { inflamación y recesión gingival }\end{array}$ & Falta de conocimiento \\
Rotura de la cabeza del tornillo de fijación & &
\end{tabular}

Tabla II. Ventajas y desventajas de miniplacas en comparación con minitornillos.

\begin{tabular}{ll}
\hline Ventajas: & Desventajas*: \\
\hline Permiten la colocación alejados de las raíces de los dientes & $\begin{array}{l}\text { El procedimiento de colocación implica colgajo } \\
\text { mucoperióstico } \\
\text { Puede generar irritación en la mejilla y labios }\end{array}$ \\
$\begin{array}{l}\text { Presentan mayor estabilidad tridimensional } \\
\text { No necesitan de gran colaboración del paciente }\end{array}$ & $\begin{array}{l}\text { Cicatrización de tejidos blandos demora } 7 \text { a } 10 \\
\text { días }\end{array}$ \\
$\begin{array}{ll}\text { Permite el uso de mayores fuerzas (mayor valor del anclaje) y e I } \\
\text { Pector de fuerza pasa por el centro de resistencia del maxilar. }\end{array}$ & $\begin{array}{l}\text { mismo acto quirúrgico a colgajo realizado para la } \\
\text { instalación. (riesgo de intervenciones adicionales } \\
\text { en caso de rotura o movilidad) }\end{array}$ \\
Muy baja tasa de falla & Mayor probabilidad de infección
\end{tabular}

Mayor avance maxilar

Menor rotación antihoraria del maxilar

Elásticos intermaxilares pueden utilizarse 24 horas al día

Mayor mejora del perfil facial

Los cambios verticales no se reflejan en las estructuras

craneofaciales.

Mayor mejora en overjet y relación molar.

Se pueden utilizar en pacientes de mayor edad para lograr avance maxilar, en comparación con el anclaje dental .

Menor rotación horaria de la mandíbula. 


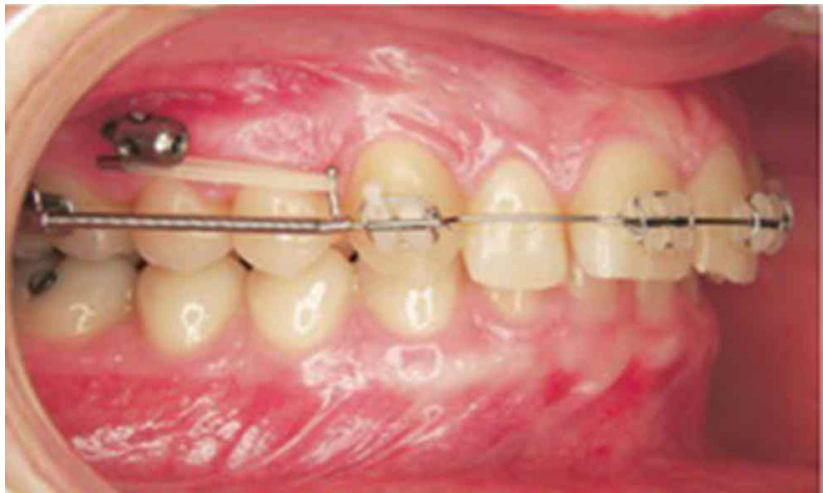

Fig. 1. Distalización molar con elasticos entre miniplacas y hook adyacente a coil cerrado hasta el tubo. Inicialmente no se cementó brackets en los premolares.

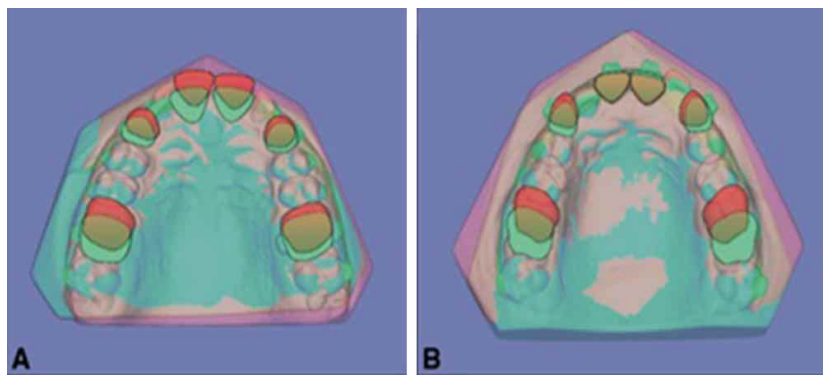

Fig. 2. Modelos maxilares, antes (rojo) y después (verde) de la distalización. Nótese el aumento del ancho intercanino y la distalización de premolares. A. Disminución de Overjet en el grupo son acople anterior previo. B. No reducción del overjet en el grupo que tenia buen acople anterior al inicio.

Los aumentos en los anchos intercanino e intermolar se pueden explicar parcialmente porque estos dientes se movieron a lo largo del reborde alveolar que es más ancho hacia distal, pero también por la línea de acción de fuerza ubicada por vestibular.

Los valores de distalización de toda la arcada superior obtenidos en este estudio fueron en promedio de 3,3+- 1,8 $\mathrm{mm}$ (rango, 0,3-7,8 $\mathrm{mm}$ ) en un período promedio de 7,0 meses, muestra que las miniplacas pueden ser una alternativa sencilla y efectiva en adultos. Este enfoque reduciría aún más la necesidad de extracciones en pacientes con apiñamiento o protrusión incisiva.

Por otro lado, en casos en que se requiere de extracciones para corregir la maloclusión clase II o biprotrusión, podemos ver en un estudio realizado el 2008 (Lai et al., 2008) que muestra la efectividad de la retracción en masa mediante anclaje esqueletal se obtuvieron diferencias significativas en los movimientos de los dientes entre los grupos de fuerza extraoral y mini-implante (minitornillos o miniplaca). Ambos gru- pos de anclaje esquelético tuvieron una mayor retracción del incisivo $(6,9 \mathrm{~mm}$ para el minitornillo, 7,3 mm para la miniplaca) que el grupo de fuerza extraoral $(5,5$ $\mathrm{mm}$ ). Además, los dientes posteriores maxilares en los pacientes tratados con miniplacas mostraron una mayor intrusión que aquellos que recibieron anclaje con minitornillos.

Mediante el uso de anclaje esqueletal con miniplacas se logra una mayor retracción de los dientes anteriores maxilares, una menor pérdida de anclaje de los dientes maxilares posteriores y la posibilidad de intrusión molar maxilar.

\section{CORRECCIÓN ORTOPÉDICA DE MALOCLUSIÓN CLASE II}

La maloclusión de clase II es una de las condiciones más frecuentes en ortodoncia y puede ser causado por protrusión maxilar, retrusión mandibular, o una combinación de ambos. Entre estas condiciones la retrusión mandibular es la más común (Ozbileket al., 2017).

Aunque la eficacia de los aparatos funcionales removibles y fijos ha sido demostrada, son muchos los efectos secundarios desfavorables, una desventaja principal de los aparatos funcionales fijos es la inclinación a mesial de la dentición mandibular y la protrusión de los incisivos inferiores, esta situación causa una corrección temprana del overjet y limita la corrección esquelética, al combinar minitornillos, con un dispositivo como Forzus no evitarían los efectos secundarios (Unal et al., 2015) pero en si usamos combinación Forzus con miniplacas se pueden ver resultados favorables (Unal et al.; Celikoglu et al., 2016; Turkkahraman et al., 2016; Ozbilek et al.) (Fig. 3).

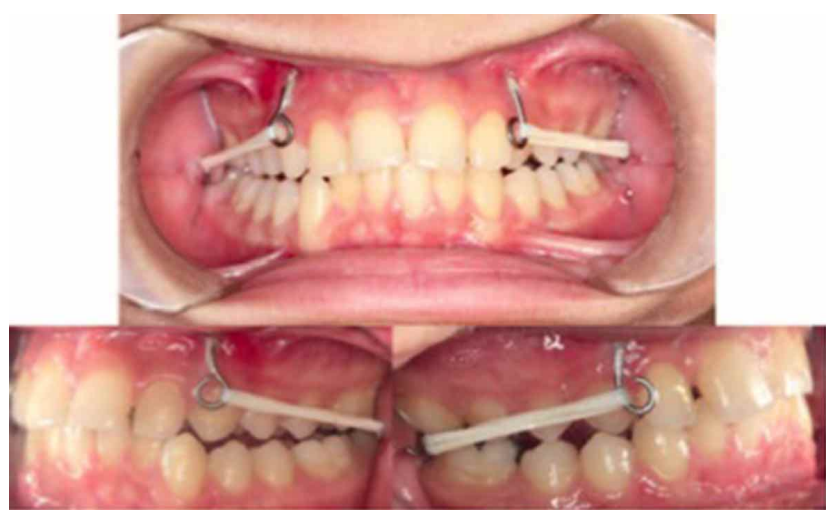

Fig. 3 Tratamineto de clase II con elásticos fijados a miniplacas. 
En estos estudios los aparatos se usaron hasta que se logró una clase I canina, molar y se disminuyó el overjet (Unal et al.; Celikoglu et al., 2016; Ozbilek et al.).

En cuanto a los resultados, se informó que este nuevo enfoque fue eficaz para corregir la maloclusión de clase II, sin producir protrusión de los incisivos mandibulares y lograr mejoras esqueletales (Unal et al.).

Celikoglu et al. (2016) compararon el efecto del Forsus anclado a miniplacas, y del Herbst, observándose cambios esqueléticos y en los tejidos blandos similares en ambos grupos. El crecimiento hacia delante de los maxilares se inhibió en ambos grupos. Se incrementó el desplazamiento hacia delante y hacia abajo de la mandíbula, representado por el ángulo SNB. Esos cambios mejoraron las relaciones maxilomandibulares en los grupos Herbst y Forsus anclados esqueléticamente.

Ambos aparatos causaron un ligero aumento en el ángulo SN-GoGn, el labio superior retrocedió y el pogonion blando y labio inferior se movieron hacia adelante y hacia abajo en ambos grupos, contribuyendo a los cambios esqueléticos y dentoalveolares.

La principal diferencia entre ellos se encontró en las inclinaciones de los incisivos superiores e inferiores, en el grupo de Forsus con anclaje esquelético, los incisivos superiores se retruyeron significativamente más que el grupo del Herbst, y se encontró que los incisivos mandibulares se retruyeron en el grupo del Forsus con anclaje esquelético, posiblemente debido a la presión de los incisivos superiores y el labio inferior.

El vector de fuerza anterior y descendente del dispositivo estimuló significativamente el crecimiento mandibular, con un movimiento hacia delante, y, por lo tanto, se logró la corrección de la maloclusión esquelética de clase II y la convexidad del perfil. El aumento significativo en la longitud mandibular efectiva (Turkkahraman et al.; Celikoglu et al., 2014). La postura hacia adelante de la mandíbula, modificada por los aparatos, aumenta el crecimiento condilar verticalmente y aumenta la altura facial posterior y anterior (Turkkahraman et al.).

Según Turkkahraman et al., quienes compararon la efectividad del Forsus con y sin anclaje esqueletal, se determinó que el aumento casi del $50 \%$ más de la longitud mandibular con el anclaje de miniplaca, lo que se puede explicar por ser una unidad de anclaje más estable, de menos pérdida, y porque las fuerzas son aplicadas directamente a las bases óseas y no en las piezas dentarias (Ozbilek et al.).

Los cambios dentoalveolares fueron la distalización e intrusión de los molares maxilares, pudiendo causar extrusión y retrusión de los incisivos superiores debido al arco pesado que conecta ambos segmentos, y también puede deberse a las fuerzas dirigidas hacia atrás y hacia arriba que actúan directamente sobre los molares maxilares.

Se observó una retrusión significativa de los incisivos inferiores efectos que combinados con los esqueletales causaron una corrección significativa en el overjet, y aumento del overbite (Celikoglu et al., 2014; Turkkahraman et al.; Ozbilek et al.).

Al-Dumaini et al. (2018) quisieron comparar pacientes sin tratamiento con pacientes con anomalías esqueletales clase II y miniplacas de anclaje esquelético bimaxilares. (Fig. 4)

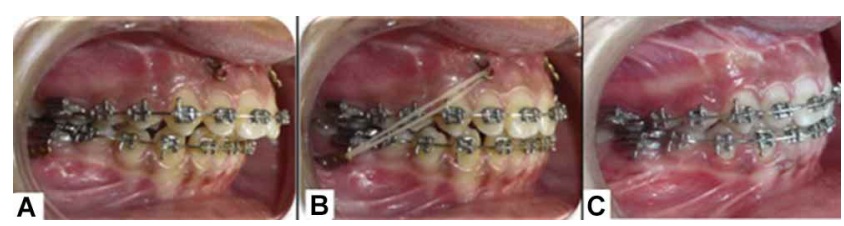

Fig. 4. Miniplacas Luego del Periodo de Cicatrización. B. Aplicación de elástico intermaxilar. C. Clase I canina y molar.

El maxilar fue afectado positivamente por la restricción de crecimiento hacia Adelante. Su largo disminuyó $1,18 \mathrm{~mm}$ y la posición maxilar fue retruída (SNA, $\left.1.65^{\circ}\right)$. La mandíbula aumentó su largo como resultado del incremento en largo de la rama $(2,03 \mathrm{~mm})$ y cuerpo $(1,2 \mathrm{~mm})$. Los cambios de ambos maxilares resultaron en una reducción significativa de la convexidad del perfil esqueletal de $5,35^{\circ}$.

La rotación antihoraria del plano mandibular se dio como resultado del movimiento de la mandíbula hacia arriba y hacia adelante. Se logró una ligera inclinación hacia lingual de los incisivos inferiores en los pacientes con miniplacas.

Estos resultados confirman que el tratamiento bimaxilar basado en miniplacas promueve la corrección de las anomalías esqueletales clase II de manera esqueletal y no a través de cambios dentoalveolares (Al-Dumaini et al.). 
En cuanto a la estética facial, el pogonion blando avanzó significativamente mejorando la convexidad del tejido blando facial, se observó una importante retrusión del labio superior y una ligera retrusión del labio inferior, lo que podría estar relacionado con la inclinación post tratamiento de los incisivos inferiores (Celikoglu et al., 2014; Unal et al.; Turkkahraman et al.; Ozbilek et al.).

\section{INTRUSIÓN POSTERIOR: MORDIDA ABIERTA}

Se han sugerido varios tratamientos de ortodoncia para la mordida abierta (MA), que implican fuerzas extrusivas en los dientes anteriores o fuerzas intrusivas en los dientes posteriores.

En los últimos años, los dispositivos de anclaje temporal (TAD) se han utilizado cada vez más en los pacientes con MA esqueletal porque proporcionan una intrusión molar absoluta. Además, con el uso de TAD,

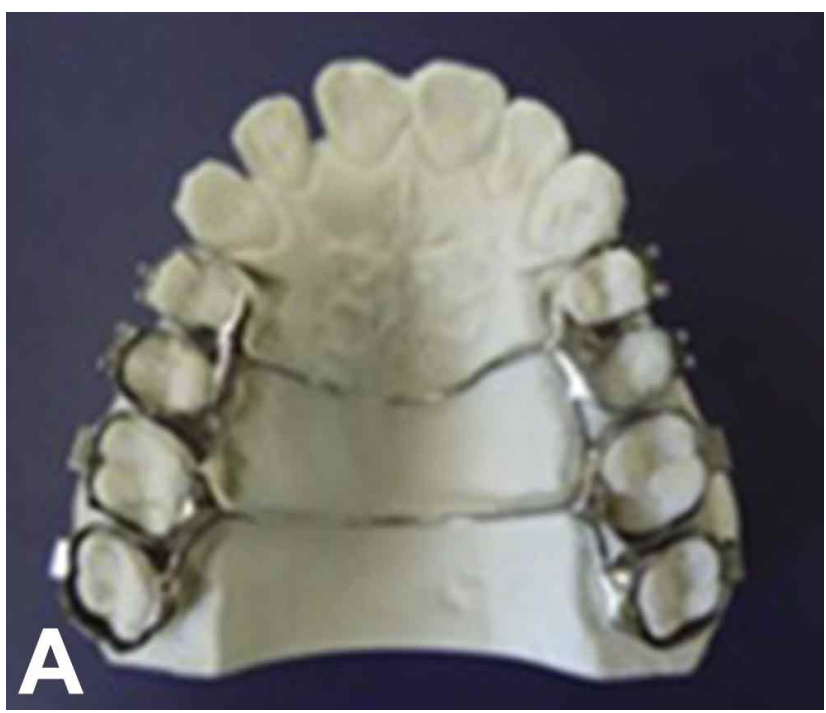

Fig. 5. Sistema de instrucción con BTP doble y miniplacas. se ha hecho posible la corrección vertical de la región dentoalveolar posterior sin efectos secundarios desfavorables, como la extrusión de las piezas anteriores.

El pilar de la cresta infracigomática se ha utilizado con éxito para proporcionar un anclaje esquelético (Reichert et al., 2014; Turkkahraman et al.).

Numerosos informes de casos han demostrado que, al menos a corto plazo, en el maxilar o la mandíbula, las miniplacas ayudan a la intrusión de los molares superiores e inferiores de hasta $3-5 \mathrm{~mm}$, mientras que también logran la rotación en sentido antihorario de la mandíbula. Este tratamiento permite al ortodoncista cerrar las MA sin extruir los dientes anteriores, que son propensos a la recidiva y a la reabsorción radicular.

En un estudio realizado el 2016 donde se realizó intrusión desde premolares hasta segundos molares. Se unieron a las miniplacas a través de cadenetas o resortes helicoidales de niti (Fig. 5).

En todos los estudios mostrados en la Tabla III se logró la intrusión de los molares superiores en un periodo de 5 a 8 meses de tratamiento.

En cuanto a la recidiva, podemos verlo resumido en la Tabla IV.

Este método es una alternativa segura, rápida y menos costosa a la cirugía ortognática, sin embargo, es una técnica relativamente nueva y hasta la fecha sigue habiendo una falta de evidencia de estabilidad a largo plazo del cierre de la MA con TAD, se deben realizar más estudios en para determinar la estabilidad y eficacia a largo plazo de este método como tratamiento (Reichert et al.; Dadgar et al., 2017).

Tabla III. Comparación de estudios donde se realizó cierre de mordida abierta y sus consecuencias a nivel de ortodoncia.

\begin{tabular}{|c|c|c|c|c|}
\hline $\begin{array}{l}\text { Factores a evaluar } \\
\text { Intrusión molar }\end{array}$ & $\begin{array}{l}\text { Turkkahraman et al., } 2016 \\
3,59 \mathrm{~mm}\end{array}$ & $\begin{array}{l}\text { Marzouk et al., } 2016 \\
3.04 \mathrm{~mm}\end{array}$ & $\begin{array}{l}\text { Oliveira et al.,2015 } \\
2,03 \mathrm{~mm}\end{array}$ & $\begin{array}{l}\text { Eiman \& Kassem, } 2018 \\
3 \mathrm{~mm}\end{array}$ \\
\hline $\begin{array}{l}\text { Consecuencias } \\
\text { Ortodónticas }\end{array}$ & $\begin{array}{l}\text { Rotación anterior mandibular, } \\
\text { cierre del plano mandibular en } \\
2,25^{\circ} \text {, Mentón hacia arriba y hacia } \\
\text { delante en } 3 \mathrm{~mm} \text {. Menor altura } \\
\text { facial anteroinferior. Perfil facial } \\
\text { equilibra do }\end{array}$ & $\begin{array}{l}\text { Movimiento antihorario } \\
\text { mandibular. } \\
\text { Movimiento del } \\
\text { mentón, punto B y } \\
\text { pogonion hacia } \\
\text { adelante y arriba. } \\
\text { Menor ángulo ANB, } \\
\text { ángulo del plano } \\
\text { mandibular y altura } \\
\text { facial anterior. Menor } \\
\text { convexidad del tejido } \\
\text { blando facial, mejora } \\
\text { del perfil. }\end{array}$ & $\begin{array}{l}\text { Sin cambios en el } \\
\text { ángulo del plano } \\
\text { palatino ni en el } \\
\text { posicionamiento } \\
\text { anteroposterior de los } \\
\text { molares. Menor } \\
\text { ángulo del plano } \\
\text { mandibular y altura } \\
\text { facial inferior. } \\
\text { Rotación antihorario } \\
\text { mandibular. }\end{array}$ & $\begin{array}{l}\text { Movimiento hacia } \\
\text { adelante de pogonion } \\
\text { duro y blando, al igual } \\
\text { que mentón duro y } \\
\text { blando. Menor altura } \\
\text { facial. Movimiento del } \\
\text { labio inferior hacia } \\
\text { adelante. }\end{array}$ \\
\hline
\end{tabular}


Tabla IV. Comparación de recidiva molar y de overbite en distintos estudios en un promedio total de 20 meses.

\begin{tabular}{lcccc}
\hline Parámetros a evaluar & Sugawara et al. & Lee y Park & Baek et al. & Scheffler et al. \\
\hline Recidiva molar & $27,2 \%-30 \%$ & $10,36 \%$ & $22,88 \%$ & $16 \%-22 \%$ \\
Recidiva Overbite & $(12$ meses $)$ & $(17,4$ meses $)$ & $(36$ meses $)$ & $(12-24$ meses $)$ \\
\hline
\end{tabular}

La ausencia de un método estandarizado de intrusión, las medidas de resultado y las diferencias en los protocolos seguidos para la intrusión molar (en un arco o en ambos arcos) han conducido a la conclusión de una evidencia clínica débil.

\section{CORRECCIÓN ORTOPÉDICA DE CLASES III}

Las clases III esqueletales pueden ser consecuencia de una deficiencia maxilar y/o un prognatismo mandibular, por lo tanto, es necesario un análisis del crecimiento mandibular y maxilar como prerrequisito para un tratamiento ortopédico adecuado. El tratamiento ortopédico tiene como objetivo crear una condición fisiológica apropiada removiendo obstáculos para llevar el crecimiento en el camino correcto, este fue el concepto original de Delaire que introdujo el uso de la máscara de Delaire en los años 70 para el tratamiento de las clases III cuya causa principal era la deficiencia maxilar (De Clerck et al., 2009; De Clerck \& Swennen, 2011; Sahin et al., 2016). El objetivo del uso de la máscara es modificar el crecimiento facial aplicando fuerzas ortopédicas sobre los dientes transmitiéndolos a la base esqueletal maxilar y mandibular, y así estimular el crecimiento maxilar a nivel de las suturas y restringir o redirigir el crecimiento mandibular (De Clerck et al., 2009; De Clerck \& Swennen; Dadgar et al.), esto tendrá como efectos no deseado la extrusión y movimiento mesial de molares maxilares, proinclinación de incisivos superiores y retroinclinación de incisivos inferiores.

Es por esto, que se han propuesto el uso de miniplacas como anclaje esqueletal, ya que han demostrado ser un medio de anclaje rígido confiable para la tracción maxilar para evitar el uso de la dentición y así lograr una corrección verdaderamente esqueletal sin los efectos adversos producidos por el uso de máscara de tracción convencional.

Terapia de Máscara de Tracción con Anclaje Esqueletal. Propuesto por primera vez por Kircelli y cols en un reporte de caso de una paciente de 11 años, clase III esqueletal severa por hipoplasia maxilar con hipodoncia. Se decide tratar de aprovechar el crecimiento sutural aplicando fuerza extraoral con una máscara de tracción vía anclaje esqueletal rígido ubicados en el maxilar (Kircelli et al., 2006).

Las miniplacas fueron ubicadas en el pilar cigomatomaxilar y la pared nasal lateral del maxilar (Fig. 6). Se prefirió la pared nasal lateral del maxilar debido a su ubicación anterior a todas las suturas circunmaxilares (para estimular un crecimiento hacia abajo y anterior del maxilar) y también su ubicación anterior con respecto al centro de resistencia del maxilar (reborde posterosuperior de la fisura pterigomaxilar) (Kircelli et al.; Kaya et al., 2011; Sar et al., 2011, 2014).

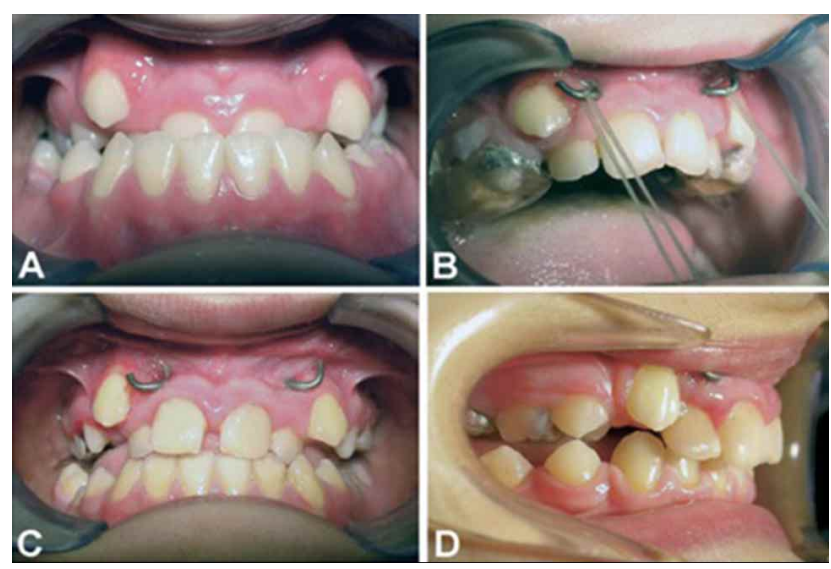

Fig. 6. A) Fotografía intraoral inicial. B) Aparato Hyrax con bite block para eliminar la interferencia incisiva y uso de elastico en las extensiones de la miniplca; C). Expansión y remoción de biteblock despues de la corrección del overejet negativo; D). Continua tracción activa hasta que se logra un overjet positivo de $5 \mathrm{~mm}$.

La tasa de éxito en la pared nasal lateral es de 93 a 95\% (Sar et al., 2011, 2014).

En la Tabla $V$ se resumen los cambios obtenidos en estos estudios (Kircelli et al.; Kircelli \& Pektas, 2008; Sar et al., 2011, 2014).

Bozkaya et al. (2017) quisieron evaluar el efecto de la terapia con máscara facial y anclaje al pilar zigomático usando dos miniplacas. Esto lo realizó en 18 pacientes clase III esqueletal (10 niñas y 8 niños de edad promedio $11,4 \pm 1,28$ años) comparados con 18 pacientes control ( 9 niñas y 9 niños de edad promedio $10,6 \pm 1,12$ años). 
Tabla V. Resumen de los cambios positivos cefalométricos, dentarios, esqueléticos y de perfil blando gracias a la máscara de tracción y miniplacas a nivel del pilar nasal.

\begin{tabular}{ll}
\hline Zona a evaluar & Cambios luego del tratamiento \\
\hline Maxilar & Avance y rotación antihoraria \\
Mandíbula & $\begin{array}{l}\text { Rotación horaria, punto B y pogonion movimiento hacia posterior, } \\
\text { apertura del ángulo mandibular y mejora en relación maxilo-mandibular }\end{array}$ \\
Dimensión vertical & Aumento \\
Altura facial inferior y total & Aumento \\
Overjet & Aumento \\
Tercio medio & Avance \\
Perfil & Favorable \\
\hline
\end{tabular}
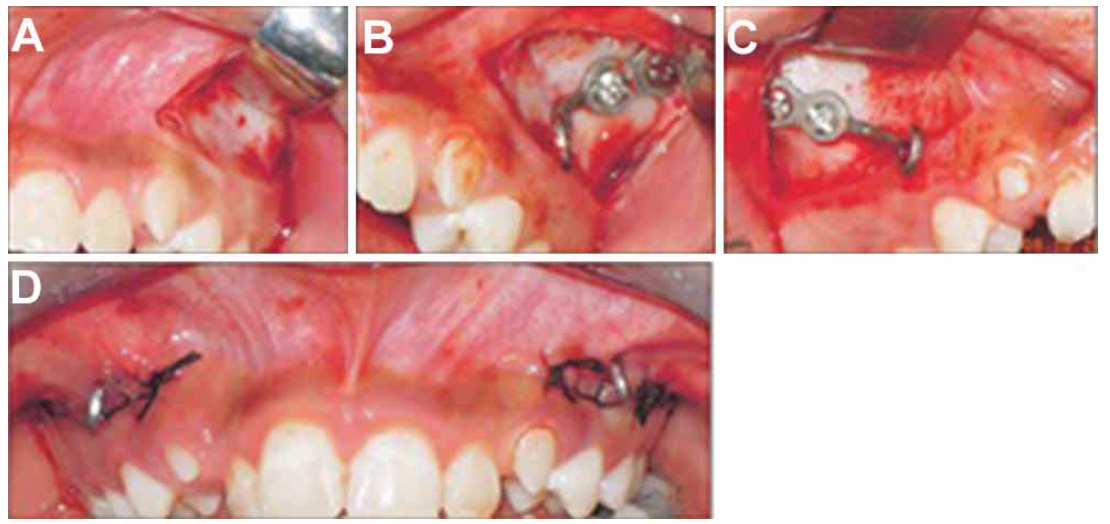

Fig. 7. Miniplacas amoldadas para adaptase a las estructuras anatomicas. Se usaron dos mini tornillos autoperforantes.

Tabla VI. Resumen de los cambios positivos cefalométricos, dentarios, esqueléticos y de perfil blando gracias a la máscara de tracción y miniplacas a nivel del pilar cigomático.

\begin{tabular}{|c|c|}
\hline Zona a evaluar & Cambios luego del tratamiento \\
\hline Maxilar & Protracción y avance \\
\hline Mandibula & $\begin{array}{l}\text { Rotación hacia abajo y atrás. Punto B movimiento hacia } \\
\text { posterior, mejora en relación maxilo mandibular }\end{array}$ \\
\hline Altura facial inferior & Aumentó \\
\hline Overjet & Aumentó \\
\hline Overbite & Disminuyó \\
\hline Perfil & $\begin{array}{l}\text { Favorable (protrusión labio superior y retrusión labio } \\
\text { inferior) }\end{array}$ \\
\hline
\end{tabular}

Se colocaron en el pilar cigomático por estar cerca del centro de resistencia del complejo nasomaxilar y por tener suficiente grosor óseo para insertar la miniplaca (Fig. 7). En el grupo en tratamiento se obtuvieron las conclusiones resumidas en la Tabla VI.

Tracción Maxilar Con Elásticos De Clase III y Miniplacas (BAMP). Con el objetivo de reducir los efectos dentoalveolares del uso de máscara de tracción frontal con aparatos intraorales y de mejorar la cooperación del paciente eliminando el uso del aparato extraoral, De Clerck introdujo en el 2009 la terapia de tracción maxilar con elásticos de clase III anclados a miniplacas en ambos maxilares.

De Clerck planteó que el uso de fuerzas contínuas moderadas en vez de fuerzas altas interrumpidas puede tener una respuesta más favorable del crecimiento maxilar y además el uso de elásticos maxilomandibular es menos limitante en términos sociales, por lo tanto, se pueden usar fácilmente las 24 horas del día (De Clerck et al., 2009).

Una revisión sistemática realizada por Rodríguez de Guzmán-Barrera et al. (2017) compararon pacientes con 4 miniplacas versus mascara facial. El valor de WITS cambio significativamente aumentando $1,28 \mathrm{~mm}$ y el ángulo SNA por $0,60^{\circ}$. Concluyendo que en pacientes clase III las miniplacas presentan mayores resultados en cuanto a tracción (Van Hevele et al., 2018). 


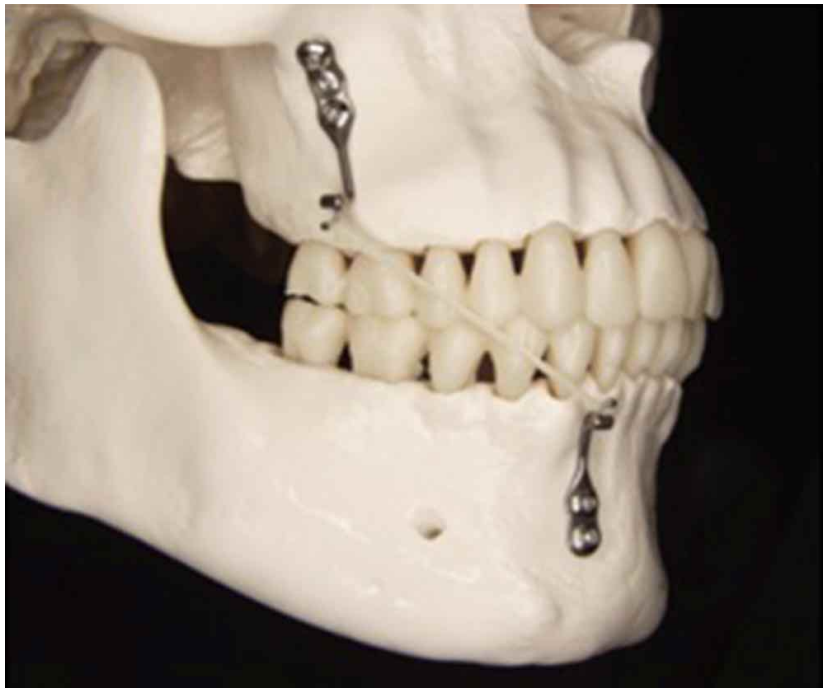

Fig. 8. Ubicación miniplacas para el protocolo BAMP. blando. Estos dos estudios hallaron los siguientes datos preliminares (De Clerck et al., 2009, 2010).

Cefalometricamente hubo cambios en el maxilar de un $60 \%$. Un avance significativo como un todo y rotación antihoraria del maxilar en uno 1 de los casos. En la mandíbula hubo cambio en un $40 \%$. No se observó rotación de la mandibula en 2 de los 3 casos. Efectos restrictivos en la mandíbula en pto. B y $P$. e incremento en longitud mandibular pero menor que un grupo no tratado. El perfil tuvo cambios favorables (protrusión labio superior y retrusión labio inferior, no se desplaza el mentón). Aumento de ANB, Witts y de la convexidad facial. En los incisivos inferiores hubo proinclinación y descompensación del tipping lingual de los incisivos mandibulares. En cuanto al overjet, hubo corrección de la mordida invertida.

Tabla VII. Resumen de los cambios positivos cefalométricos, esqueléticos y de tejido blando gracias a la Tracción Maxilar con Elásticos de Clase III y Miniplacas en estudio tridimensional.

\begin{tabular}{|c|c|c|c|}
\hline Estructuras a evaluar & Cambios cefalométricos & Cambios esqueléticos & Cambios tejido blando \\
\hline Maxilar & & $\begin{array}{l}\text { Protracción maxilar y cigomática } \\
\text { con mínima rotación antihoraria } \\
*\end{array}$ & $\begin{array}{l}\text { Cambios significativos, aunque } \\
\text { solo evidentes en labio } \\
\text { superior. Desplazamiento } \\
\text { hacia anterior de todos los } \\
\text { puntos. }\end{array}$ \\
\hline Mandíbula & & $\begin{array}{l}\text { Compensa el crecimiento } \\
\text { mandibular. Mejora en la } \\
\text { discrepancia esqueletal. } \\
\text { Rotación antihoraria por cierre } \\
\text { del ángulo goniaco. }\end{array}$ & Mejora el perfil facial. \\
\hline Cóndilo & & Se mueven posteriormente. & \\
\hline Rama & & $\begin{array}{l}\text { Desplazamiento distal de la } \\
\text { rama posterior. Minimiza el } \\
\text { efecto de aumento de overbite y } \\
\text { disminuye la proyección del } \\
\text { mentón. }\end{array}$ & \\
\hline Borde Mandibular & & $\begin{array}{l}\text { Mayoría de los pacientes se } \\
\text { observa un desplazamiento } \\
\text { paralelo sin rotación. }\end{array}$ & \\
\hline Fosa Glenoídea & & $\begin{array}{l}\text { Se remodela con una } \\
\text { reabsorción de eminencia } \\
\text { posterior y aposición de } \\
\text { eminencia anterior. Correlación } \\
\text { entre el desplazamiento } \\
\text { posterior del cóndilo con la } \\
\text { cantidad de aposición y } \\
\text { reabsorción ósea a nivel de la } \\
\text { fosa glenoídea. }\end{array}$ & \\
\hline Mentón & & $\begin{array}{l}\text { Mantuvo posición anterposterior, } \\
\text { con un rango de respuesta } \\
\text { variable. Contibuye a un } 40 \% \text { de } \\
\text { la mejora en la discrepancia } \\
\text { maxilo-mandibular. Mayor } \\
\text { proyección. }\end{array}$ & $\begin{array}{l}\text { Mentón blando mostró cambios } \\
\text { de posición similar al punto } \\
\text { mentón. }\end{array}$ \\
\hline $\begin{array}{l}\text { Ángulo Goniaco } \\
\text { Ángulo del plano } \\
\text { mandibular }\end{array}$ & $\begin{array}{l}\text { Cierre } \\
\text { Cierre (Produce un } \\
\text { desplazamiento vertical } \\
\text { mayor en gonion en } \\
\text { comparación a mentón }\end{array}$ & For & $\begin{array}{l}\text { Cambio en el tejido blando. } \\
\text { Cambios significativos, aunque } \\
\text { solo evidentes en el labio } \\
\text { superior. Desplazamiento } \\
\text { previo de todos los puntos. }\end{array}$ \\
\hline Overbite & & Aumenta & \\
\hline
\end{tabular}


También se han realizado estudios tridimensionales para evaluar los cambios maxilares y mandibulares como efecto de este protocolo mostrados en la Tabla VII.

Que haya protracción maxilar y zigomática con mínima rotación antihoraria coincide con la rotación antihoraria maxilar mínima encontrada el estudio 2D de De Clerck et al. (2010). Este hallazgo se contradice con la definición hipotética de Teuscher de la posición del centro de resistencia del maxilar en la biomecánica de desplazamiento óseo. Si el centro de resistencia está ubicado en el pilar maxilar, la línea de fuerza de tracción de BAMP al centro de resistencia resultaría en una marcada rotación antihoraria del maxilar. La mínima rotación del maxilar sugiere que el centro de resistencia del tercio medio esta probablemente ubicado posterior e inferior al definido por Teuscher (Nyugen et al., 2011).

Otro hallazgo interesante fue la apertura de las suturas cincunmaxilares que fue evidente en algunos pacientes. El desplazamiento anterior del maxilar se observó sin desarticulación de las estructuras faciales, contrario a otros protocolos de MTF, el protocolo de BAMP no realiza un EPR antes de la tracción (Nyugen et al., 2011).

La sutura palatina transversal ha mostrado tener la mayor apertura de todas las suturas circunmaxilares después de la tracción en primates. Estos hallazgos fueron confirmados en este estudio (Nyugen et al., 2011).

Ahora, en cuanto a la gran variación de la posición del mentón, esto puede ser explicado por 4 factores que ocurren simultáneamente y determinan el patrón individual de crecimiento mandibular y su respuesta al tratamiento (Nyugen et al., 2014). Estos son: Cantidad y dirección del crecimiento condilar, remodelado óseo en la fosa articular, rotación horaria o antihoraria de la mandíbula y la cantidad de cierre o apertura del ángulo goníaco.

Estudios anteriores en pacientes clases III esqueletal del mismo rango etáreo, determinaron que el aumento en longitud mandibular es de $3 \mathrm{~mm}$ por año y este se mantiene casi igual con el tratamiento ortopédico de tracción maxilar. Esta diferencia encontrada en la proyección del mentón no puede ser explicada por restricción del crecimiento mandibular (De Clerck et al., 2012).
Segundo, el desplazamiento posterior del cóndilo se produce manteniendo el ancho condilar y con un remodelamiento de la fosa articular. Aunque este desplazamiento del cóndilo puede contribuir al desplazamiento posterior de toda la mandíbula, la magnitud de desplazamiento no es suficiente para explicar la restricción de la proyección anterior del mentón. Además, no se encontró una correlación entre el desplazamiento del mentón con el desplazamiento condilar ni la remodelación en la fosa articular (De Clerck et al., 2012).

Por último, los bordes posteriores de la rama se desplazaron hacia posterior al igual que el mentón, como el desplazamiento de la rama fue menor que el desplazamiento condilar y sin rotación horaria de la mandíbula este resulta en un cierre del ángulo goníaco. Este desplazamiento posterior de la rama mandibular con cierre del ángulo goníaco son responsables de la mantención de la proyección del mentón (De Clerck et al., 2012).

Agregado a esto, el estudio tridimensional realizado por Nguyen et al. (2014) encontraron 3 patrones de desplazamiento condilar hacia abajo y atrás, posterior y por último arriba y atrás.

Aquellos pacientes que tenían un desplazamiento hacia arriba y atrás tenían un ángulo goníaco mayor al inicio del tratamiento, en estos pacientes el vector de fuerza probablemente estaba dirigida bajo el centro de resistencia de la mandíbula a diferencia de los otros 2 patrones de desplazamiento (Nyugen et al., 2014).

En resumen, la restricción del desplazamiento anterior del mentón se obtiene por una combinación de desplazamiento posterior de la rama y cierre del ángulo goníaco donde se afecta la forma mandibular más que el tamaño mandibular (De Clerck et al., 2012; Nyugen et al., 2014). No hubo rotación posterior de la mandíbula. Existe una alta correlación entre el remodelamiento anterior y posterior de la fosa glenoidea y el desplazamiento del cóndilo.y una gran variabilidad interindividual en la respuesta al tratamiento. Luego de realizar el protocolo BAMP se observa mantención de la corrección de la clase III en un seguimiento de 11 a 38 meses después (De Clerck et al., 2009).

En cuanto a cuando comenzar el tratamiento según Delaire, McNamara y Proffit para lograr efectos esqueletales en vez de dentales se recomienda ini- 
ciar tratamiento a una edad temprana, antes de los 8 años (Kircelli \& Pektas). Esto se debe a que la morfología sutural de la región palatomaxilar, se vuelve más interdigitada con la edad, por lo que es más difícil de desarticular el hueso palatino del proceso pterigoideo. Cha investigo los cambios esqueletales y dentoalveolares, y encontró que sujetos pasado el peak de crecimiento puberal, disminuyen el efecto de avance maxilar esqueletal en un $60 \%$ y efecto dentoalveolar aumenta en $40 \%$. Es por esto que algunos tratantes pueden elegir retrasar el tratamiento definitivo en pacientes de "mayor edad" con clase III moderada hasta que el crecimiento termine para realizar un tratamiento ortodóncico quirúrgico en vez de arriesgar crear un perfil no satisfactorio con una relación dental comprometida. En este contexto se debe aprovechar el uso anclaje esqueletal con miniplacas para lograr cambios esqueletales y de perfil (Kircelli \& Pektas).

Sin embargo, las miniplacas también tienen sus limitaciones en cuanto a edad, la instalación de miniplacas no puede ser realizada en niños muy jóvenes por la reducida altura del hueso alveolar maxilar, y porque las miniplacas mandibulares no pueden ser posicionadas antes de la erupción de los caninos, por lo tanto tracción ortopédica anclado a miniplacas no puede ser iniciada antes de los 10 años (De Clerck et al., 2009).

En cuanto al exito de las miniplacas BAMP, en comparación a minitornillos y microtornillos muestran mayor estabilidad. Sin embargo, solo se ha investigado el efecto de la estabilidad en miniplacas sometidas a fuerzas continuas. Las fuerzas generadas por elásticos intermaxilares son discontinuas en tiempo, magnitud, y dirección debido a los movimientos de la mandíbula en función (De Clerck \& Swennen).

Las tasas de éxito son de un 92,5 a $97 \%$ y las fallas ocurren normalmente en el maxilar (De Clerck \& Swennen; Van Hevele et al.).

En el estudio realizado por De Clerck \& Swennen, las miniplacas que fallaron ocurrieron en los pacientes más jóvenes, por lo tanto los autores proponen no usar miniplacas para anclaje esqueletal antes de los 11 años, debido al alto riesgo de baja calidad ósea.

CONCLUSIONES. Las miniplacas, como dispositivos de anclaje temporal (TADs), han demostrado ser efectivas, en los tratamientos de las maloclusiones expues- tas anteriormente, como son, las maloclusiones de clase II, clase III, y mordida abierta.

Las miniplacas al funcionar en base a un anclaje esqueletal y no necesitar la cooperación del paciente para su uso (a excepción de los protocolos de tratamiento en clases III y clases II, donde el uso de elásticos depende del paciente), genera fuerzas constantes y directas a la base ósea, siendo más efectivas, ya que aumenta el control general y la previsibilidad del tratamiento de ortodoncia.

En comparación con los minitornillos, tienen la ventaja de que se pueden colocar donde más se necesita el anclaje, lejos de las raíces de las piezas dentarias y por consecuencia evita la interferencia con los movimientos radiculares. Además, presentan mayor estabilidad tridimensional.

Sin embargo, tienen algunas desventajas ya que presentan áreas limitadas para su inserción, son más costosos y requieren dos procedimientos quirúrgicos mínimos (inserción/remoción).

DURÁN, F.; HORMAZÁBAL, F.; TOLEDO, X.; CHANG, R. H.; GONZÁLEZ, N. \& SCIARAFFIA, P. A general and updated description of miniplates and miniscrews. Dentoalveolar and skeletal effects. Int. J. Odontostomat., 14(1):136-146, 2020.

ABSTRACT: In orthodontics, miniplates are used as a Temporary Anchoring Device (TAD) for the purpose dental movements, allowing the use of orthopedic forces. In comparison with mini-screws, miniplates have the advantage of a very low rate of failure. Nonetheless, their removal requires the same surgical procedure as during installation, which is an obvious disadvantage. The aim of this study is to review the indications of miniplates in patients with open bite, class II and class III anomalies, and review how miniplates improved orthodontics treatments. Information was obtained by a search in PubMed with the keywords listed below. Miniplates are indicated for retraction in mass of the arcade, where it was seen that the force of $150 \mathrm{~g}$ applied on maxillary molars, is sufficient not only to push the molars back into a corrected class I, but also to initiate retraction of premolars, canines, and incisors. In open-bite patients, mini plates, are achieved as a safe method, that is quick and a less expensive alternative to orthognathic surgery. Further, in class II and III patients they are used without producing dentoalveolar effects replacing extraoral devices as facemasks, with intraoral devices and elastics. (BAMP).

KEY WORDS: orthodontic miniplate, orthodontic miniscrew, temporary anchoring device, bone anchor device, Bone Anchoring Maxillary Protraction (BAMP), skeletal anchorage. 
DURÁN, F.; HORMAZÁBAL, F.; TOLEDO, X.; CHANG, R. H.; GONZÁLEZ, N. \& SCIARAFFIA, P. Una descripción general y actualizada de miniplacas y minitornillos. efectos dentoalveolares y esqueléticos. Int. J. Odontostomat., 14(1):136-146, 2020.

\section{REFERENCIAS BIBLIOGRAFICAS}

Al-Dumaini, A. A.; Halboub, E.; Alhammdi, M. S.; Ishaq, R. A. R. \& Youssef, M. A novel approach for treatment of skeletal Class II malocclusion: Miniplates-based skeletal anchorage. Am. J. Orthod. Dentofacial Orthop., 153(2):239-47, 2018.

Baccetti, T.; De Clerck, H. J.; Cevidanes, L. H. \& Franchi, L. Morphometric analysis of treatment effects of bone-anchored maxillary protraction in growing class III patients. Eur. J. Orthod., 33(2):121-5, 2011

Bozkaya, E.; Yüksel, A. S. \& Bozkaya, S. Zygomatic miniplates for skeletal anchorage in orthopedic correction of Class III malocclusion:A controlled clinical trial. Korean J. Orthod., 47(2):118-29, 2017.

Celikoglu, M.; Buyuk, S. K.; Ekizer, A. \& Tubal, U. Treatment effects of skeletally anchored Forsus FRD EZ and Herbst appliances: A retrospective clinical study. Angle Orthod., 86(2):306-14, 2016.

Celikoglu, M.; Unal, T.; Bayram, M. \& Candirli, C. Treatment of a skeletal Class II malocclusion using fixed functional appliance with miniplate anchorage. Eur. J. Dent., 8(2):276-80, 2014.

Choi, B. H.; Zhu, S. J. \& Kim, Y. H. A clinical evaluation of titanium miniplates as anchors for orthodontic treatment. Am. J. Orthod. Dentofacial Orthop., 128(3):382-4, 2005.

Cornelis, M. A. \& De Clerck, H. J. Maxillary Maxillary Molar Distalization With Miniplates Assessed on Digital Models: A Prospective Clinical Trial. Am. J. Orthod. Dentofacial Orthop., 132(3):373-7, 2007.

Costello, B. J.; Ruiz, R. L.; Petrone, J. \& Sohn, J. Temporary Skeletal Anchorage Devices for Orthodontics. Oral Maxillofac. Surg. Clin. North Am., 22(1):91-105, 2010.

Dadgar, S.; Sobouti, F.; Armin, M. \& Esnaashari, N. Correction of Skeletal Openbite Using Zygomatic Miniplates. Br. J. Med. Med. Res., 19(6):110, 2017.

De Clerck, E. E. B. \& Swennen, G. R. J. Success rate of miniplate anchorage for bone anchored maxillary protraction. Angle Orthod., 81(6):1010-3, 2011.

De Clerck, H. J.; Cornelis, M. A.; Cevidanes, L. H.; Heymann, G. C. \& Tulloch, C. J. F. Orthopedic traction of the maxilla with miniplates: A new perspective for treatment of midface deficiency. J. Oral Maxillofac. Surg., 67(10):2123-9, 2009

De Clerck, H.; Cevidanes, L. \& Baccetti, T. Dentofacial effects of boneanchored maxillary protraction: A controlled study of consecutively treated class III patients. Am. J. Orthod. Dentofacial Orthop., 138(5):577$81,2010$.

De Clerck, H.; Nyugen, T.; de Paula, L. K. \& Cevidanes, L. Three-dimensional assessment of mandibular and glenoid fossa changes after boneanchored Class III intermaxillary traction. Am. J. Orthod. Dentofacial Orthop., 142(1):25-31, 2012.

Erverdi, N. \& Üsümez, S. Anclaje Óseo: Un Nuevo Concepto en Ortodoncia. En: Nanda, R. (Ed.). Dispositivos de Anclaje Temporal en Ortodoncia. Medellín, Amolca, 2010. pp.342-5.

Faber, J.; Morum, T. F. A.; Leal, S.; Berto, P. M. \& Carvalho, C. K. S. Miniplacas permitem tratamento eficiente e eficaz da mordida aberta anterior. Rev. Dent. Press Ortodon. Ortop. Facial, 13(5):144-57, 2008.

Kaya, D.; Kocadereli, I.; Kan, B. \& Tasar, F. Effects of facemask treatment anchored with miniplates after alternate rapid maxillary expansions and constrictions; A pilot study. Angle Orthod., 81(4):639-46, 2011

Kircelli, B. H. \& Pektas, Z. O. Midfacial protraction with skeletally anchored face mask therapy: A novel approach and preliminary results. Am. J. Orthod. Dentofacial Orthop., 133(3):440-9, 2008.

Kircelli, B. H.; Pektas, Z. O. \& Uçkan, S. Orthopedic protraction with sketeletal anchorage in patient with maxillary hypoplasia amd hypodontia. Angle Orthod., 76(1):156-63, 2006

Lai, E. H. H.;Yao, C. C. J.; Chang, J. Z. C.; Chen, I. \& Chen, Y. J. Threedimensional dental model analysis of treatment outcomes for protrusive maxillary dentition: Comparison of headgear, miniscrew, and miniplate skeletal anchorage. Am. J. Orthod. Dentofacial Orthop., 134(5):636$45,2008$.

Lama, R.; Goonewardene, M. S.; Allan. B. P. \& Sugawara, J. Success rates of a skeletal anchorage system in orthodontics: A retrospective analysis. Angle Orthod., 88(1):27-34, 2018.
Marzouk, E. S. \& Kassem, H. E. Evaluation of long-term stability of skeletal anterior open bite correction in adults treated with maxillary posterior segment intrusion using zygomatic miniplates. Am. J. Orthod. Dentofacial Orthop., 150(1):78-88, 2016.

Nagasaka, H.; Sugawara, J.; Kawamura, H. \& Kasahara T. A clinical evaluation on the efficacy of titanium miniplates as orthodontic anchorage. Orthod. Waves, 58:136-47, 1999.

Nyugen, T.; Cevidanes, L.; Cornelis, M. A.; Heymann, G.; de Paula, L. K. \& De Clerck, H. (2011). Three-dimensional assessment of maxillary changes associated with bone anchored maxillary protraction. Am. J. Orthod. Dentofacial Orthop., 140(6):790-8, 2011.

Nyugen, T.; Cevidanes, L.; Paniagua, B.; Zhu, H.; Koerich, L. \& De Clerck $\mathrm{H}$. Use of shape correspondence analysis to quantify skeletal changes associated with bone-anchored Class III correction. Angle Orthod., 84(2):329-36, 2014

Ozbilek, S.; Gungor, A. Y. \& Celik, S. Effects of skeletally anchored Class II elastics: A pilot study and new approach for treating Class II malocclusion. Angle Orthod., 87(4):505-12, 2017.

Reichert, I.; Figel, P. \& Winchester, L. Orthodontic treatment of anterior open bite: a review article--is surgery always necessary? Oral Maxillofac. Surg., 18(3):271-7, 2014.

Rodríguez de Guzmán-Barrera, J.; Sáez Martínez, C.; Boronat-Catalá, M.; Montiel-Company, J. M.; Paredes-Gallardo, V.; Gandía-Franco, J. L.; Almerich-Silla, J. M. \& Bellot-Arcís, C. Effectiveness of interceptive treatment of class III malocclusions with skeletal anchorage: A systematic review and meta-analysis. PLoS One, 12(3):e0173875, 2017.

Sahin, T.; Delforge, A.; Garreau, E.; Raoul, G. \& Ferri, J. Orthopedic treatment of Class III malocclusions using skeletal anchorage: A bibliographical review. Int. Orthod., 14(3):263-72, 2016.

Sar, C.; Özçirpici, A. A.; Uçkan, S. \& Yazici., A. C. Comparative evaluation of maxillary protraction with or without skeletal anchorage. Am. J. Orthod. Dentofacial Orthop., 139(5):636-49, 2011.

Sar, C.; Sahinog־lu, Z.; Özçirpici, A. A. \& Uçkan, S. Dentofacial effects of skeletal anchored treatment modalities for the correction of maxillary retrognathia. Am. J. Orthod. Dentofacial Orthop., 145(1):41-54, 2014.

Schätzle, M.; Mänchenn, R.; Zwahlen, M. \& Lang, N. P. Survival and failure rates of orthodontic temporary anchorage devices: a systematic review. Clin. Oral Implants Res., 20(12):1351-9, 2009.

Sekima, M. T.; de Mendonça, A. A.; Ocanha Júnior, J. M. \& Sakima, T. Sistema de Apoio Ósseo para Mecânica Ortodôntica (SAO®) miniplacas para ancoragem ortodôntica. Parte I: tratamento da mordida aberta. Rev. Dent. Press Ortodon. Ortop. Facial, 14(1):103-16, 2009.

Takaki, T.; Tamura, N.; Yamamoto, M.; Takano, N.; Shibahara, T.; Yasumura, T.; Nishii, Y. \& Sueishi, K. Clinical study of temporary anchorage devices for orthodontic treatment--stability of micro/mini-screws and mini-plates: experience with 455 cases. Bull. Tokyo Dent. Coll., 51(3):151-63, 2010.

Tsui, W. K.; Chua, H. D. P. \& Cheung, L. K. Bone anchor systems for orthodontic application: a systematic review. Int. J. Oral Maxillofac. Surg., 41(11):1427-38, 2012

Turkkahraman, H.; Eliacik, S. K. \& Findik, Y. Effects of miniplate anchored and conventional Forsus Fatigue Resistant Devices in the treatment of Class II malocclusion. Angle Orthod., 86(6):1026-32, 2016.

Unal, T.; Celikoglu, M. \& Candirli, C. Evaluation of the Effects of Skeletal Anchoraged Forsus FRD Using Miniplates Inserted on Mandibular Symphysis: ANew Approach for the Treatment of Class II Malocclusion. Angle Orthod., 85(3):413-9, 2015.

Van Hevele, J.; Nout, E.; Claeys, T.; Meyns, J.; Scheerlinck \& Politis, C. Bone-anchored maxillary protraction to correct a class III skeletal relationship: A multicenter retrospective analysis of 218 patients. J. Cranio-Maxillo-Facial Surg., 46(10):1800-6, 2018.

Dirección para correspondencia:

Francisca Durán

Cirujano Dentista UC

Santiago - CHILE

Email: fran.duranb@gmail.com

Recibido : 26-06-2019

Aceptado: 03-08-2019 